\title{
A facile extramedullary method to perform primary total knee arthroplasty in patients without hardware removal: a case series
}

\author{
Yu-Kai Tseng \\ Show Chwan Memorial Hospital https://orcid.org/0000-0001-7897-9798 \\ Yen-Nien Chen \\ Asia University \\ Chih-Han Chang \\ National Cheng Kung University Department of Biomedical Engineering \\ Kuo-An Lai \\ Kuo General Hospital \\ Chyun-Yu Yang \\ National Cheng Kung University Hospital \\ Chih-Wei Chang ( $\nabla$ u7901064@yahoo.com.tw) \\ https://orcid.org/0000-0002-7559-3264
}

\section{Research article}

Keywords: Knee arthroplasty, Retained hardware, Extramedullary, Knee

Posted Date: February 27th, 2020

DOI: https://doi.org/10.21203/rs.2.24699/v1

License: (c) (i) This work is licensed under a Creative Commons Attribution 4.0 International License. Read Full License 


\section{Abstract}

Background It is technical demanding to execute primary total knee arthroplasty (TKA) for post-traumatic knee arthritis with periarticular implants. Our purpose is to present a simple extramedullary guide technique and report the clinical results of our series of primary TKAs in patients with post-traumatic arthritis without hardware removal.

Methods Between April 2014 and March 2019, a series of seven patients with post-traumatic knee arthritis and retained hardware were included. All the surgeries were performed by the same surgeon. The mean age of the patients was 67 years. All the index procedures were performed with primary TKAs by the same surgeon according to simple extramedullary technique. The parallelism between the distal femoral and proximal tibial cuts as well as the required limb alignments were verified intraoperatively. Radiographic measurements and clinical outcomes were reviewed retrospectively.

Results Overall, there was a good restoration of postoperative mechanical alignment in all cases. The mean post-operative HipKnee-Ankle (HKA) angle was $179.4^{\circ}$. No patient required allogenic blood transfusion or prolonged hospital stays. No surgical site-specific complications were noted by chart review. In the last follow-up, no implant failure was found. Clinical assessments according to knee society score showed good knee scores (89.1), functional scores (87.9) and motion arc (112.9).

Conclusions Our results show that post-traumatic knee arthritis can be safely managed with this extramedullary method without hardware removal.

\section{Background}

Total knee arthroplasty (TKA) is an effective surgical intervention for post-traumatic knee arthritis once conservative treatment failed.[1-3] For orthopaedic surgeons, it is a great challenge as well as a technique-demanding task to perform TKAs on the post-traumatic knees with retained implants.[1] In the literatures, substantial complications like infection, stiffness, and compromised wound healing had been reported.[3,4] For technical difficulty, retained implants frequently hinder the passage of guiding rods of current intramedullary guided TKA, which may require staged removal of prior implant before the index procedure.[5] Ideals of new coding for conversion TKA were proposed due to more complicated condition for staged or simultaneous removal of implants. $[4,6]$ Even though, excessive surgical procedures prior to knee replacement predispose the later arthrofibrosis and infection.[4, 7] Simultaneous arthroplasty and removal of hardware is a choice but may increase the risks of skin necrosis, prolonged operation time, infection and periprosthetic fractures by the stress risers after the removal of prior implants.[4, 8]

In current practice, modern devices like computer-assisted navigation (CAN) or premade patient-specific block (PSB), had been reported to be of value in these complex situations without hardware removal.[5, 9] However, extra expenditure on these equipment and devices delayed their propagation and wild acceptance.[10] After reviewing related literatures about primary TKAs with retained hardware, we acknowledge that the key is to execute the required bony cuts using reliable extramedullary (EM) referencing methods.

To obtain acceptable alignments as well as surgical accuracy, the authors proposed some practical and reproducible principles for EM referencing while executing some critical bone cuts in TKAs like the proximal tibial and distal femur ones. The aim of this study was to verify the clinical effectiveness of our EM referencing method to perform primary TKAs in post-traumatic knees with retained implants.

\section{Methods}

Between April 2014 and March 2019, patients with arthritic knees and retained hardware underwent their primary TKAs by the identical surgeon using similar cemented prostheses with posterior-stabilized design (U2 knee®; United Orthopedic Co., Taiwan; Genesis II ; Smith \& Nephew New Zealand; Scorpio NRG®; Stryker; NJ, USA) were included. In these surgeries, the same EM referencing method rather than conventional intramedullary cutting jig was used due to the obstructed medullary canal by the prior orthopaedic hardware. These cases were reviewed after Institutional Review Board approval was obtained [A-ER-106-021]. 
Similar to other primary TKAs, standard radiographs including the anterior-posterior, lateral views of the knee and the longstanding scanogram, were obtained before surgery. In addition to assess the preoperative mechanical axis and angular deformity, the potential hardware hindering the bony preparation or implantation were identified within the region between the proximal tibia and distal femur cuts drawn on the images [Figure 1].

Under general anesthesia, all the operated knees were approached via the identical medial skin incision and parapatellar arthrotomy in knee flexion. A pneumatic tourniquet ranging $280 \sim 320 \mathrm{mmHg}$ was used to obtain the bloodless field during surgery. After joint exposure, both tibial and femoral osteophytes are removed. Before bone cuts, partial removal of the potential hindering screws and limited contracture release were made.

For the proximal tibial cut, a horizontal line perpendicular to the tibia mechanical axis referencing tibia and surrounding landmarks was drawn on the proximal tibia (tibial plateau) using an electrocautery in knee flexion of 90 degrees (Fig. 2A). Then a horizontal osteotomy along the line was made accordingly. The following distal femoral cut was executed based on a simple geometric principle, parallelism. A parallel line referencing prior proximal tibial cut was marked on the distal femur in knee extension under an even distraction (Fig. 2B). Then, along the line, the distal femur cut was executed with a firm, steady handed oscillating saw. To avoid unwanted injuries to the neurovascular structures behind, the cut can be performed in steps. Once the saw blade proceeds half of the distal femur condyles, the remaining cut can be finished with the knee in flexion. After these two critical cuts, the overall coronal alignment of lower extremity was determined. The required parallelism as well as the even bony cuts was verified again with the extended knee in distraction or completely bony contact (Fig. 2C \&2D).

As to the remaining femoral cuts, including the anterior, posterior, and chamfering cuts, were executed using the commercial "4in-1" cutting jigs referencing the antero-posterior axis (Whiteside's line).[11] All the knee caps were resurfaced. After evaluating the implant orientation, restored mechanical axis, soft tissue balancing and deforming stresses, similar cemented prostheses with posterior-stabilized design were implanted in all knees.

After surgery, all patients were managed according to an established clinical pathway for primary TKA at our ward, including the rehabilitation protocol, the strategy of pain control and even the fluid supplements. Prophylaxis for thromboembolic events was performed using $500 \mathrm{mg}$ of intravenous lysine acetylsalicylic acid per day for the first three postoperative days, mechanical calf compression and early ambulation. Early knee motion and ambulation were encouraged in all patients even on the same day of operation. In addition to the stable wound, active knee flexion at least 90 degrees as well as stable independent ambulation composed the conforming criteria for discharge.

\section{Assessment}

At the latest follow-up at out-patient department, one author, not involved in the original surgery, evaluated the Knee Society Clinical Rating System (KSS) and range of motion for all the patient.[12] Radiographic data, hospitalization data and all postoperative data were reviewed from inpatient electronic healthcare records. Radiological assessment was carried out using a standardized protocol. Standing radiographs were obtained with the knee in maximum extension, the patella pointing forward and both hips and ankles visible on the film. (Fig. 3) The lateral radiographs were taken with the knee in $30^{\circ}$ of flexion on a radiographic film. These standard radiographs were used to determine the HKA angles. Hospitalization data include tourniquet time, mean hemoglobin drop and length of stay. Postoperative data include emergency department (ED) visits, readmissions, post-operative site-specific complications, and mortality.

\section{Results}

Within sixty months, a total of 7 severe arthritic knees with retained hardware were converted to total knee arthroplasty using one-stage surgical intervention. Demographic features and clinical results of the 7 cases are presented in the Table 1.

The mean age of these subjects including 2 males and 5 females were 67 years (range from 57 years to 76 years). Most of them presented with a varus deformity while one patient with a valgus knee. The mean pre-operative HKA angles were $171.6^{\circ}$ 
(range from $155^{0}$ to $191^{\circ}$ ). Among the 7 knees, the most interfering cases include implants at both sides (Figs. 4 and 5).

For the operation, the mean ischemic time was 93.6 minutes (range from 56 to $132 \mathrm{~min}$ ). During hospitalization, no patients required allogenic blood transfusions or prolonged stays. Mean hemoglobin drop was $3.2 \mathrm{~g} / \mathrm{dL}$. The mean duration of their inpatient stay was 6.4 days. By electronic chart review, all patients have no ED visits, readmissions and site-specific complications throughout the follow-ups. Radiographic evaluation at the latest clinic visit had no implant revisions and no signs of radiological loosening. The mean post-operative HKA angles were $179.4^{\circ}$ (range from $177^{\circ}$ to $182^{\circ}$ ). Early recoveries and improvements in the range of motion were observed at the follow-ups. The mean post-operative range of motion was $112.9^{\circ}$ (range: $105^{\circ}-120^{\circ}$ ). All the functions and knee scores improved in all patients. The mean post-operative knee scores were 89.1 (range from 83 to 93). The mean post-operative functional scores were 87.9 (range from 85 to 95).

\section{Discussion}

It is a great challenge to perform primary TKA for patients suffering post-traumatic knee arthritis, the retained implants, residual extra-articular deformity and even the compromised femoral canal, which all hinder the use of current intramedullary guiding system to obtain accurate alignment.[5] However, there is still no clear evidence that implants should be removed or retained in patients receiving TKA with periarticular hardware. Conversion TKA, defined as Primary TKA with periarticular implants removed simultaneously or in stage, was reported to have increased wound problems and readmission rate.[4, 6] TKA without hardware removal, including intra-operative navigation or patient specific instruments, were reported to be of value in these complicated scenarios. $[5,9,13,14]$ In this study, a simplified but facile extramedullary guiding method was proposed as alternative to perform primary TKAs in patients with arthritic knee and retained implant once computer-assisted devices were unavailable.

In the literature, computer-assisted techniques has been reported to achieve accurate alignment correction and showed similar results and complication rates compared to atraumatic osteoarthritis.[5] However, they are not free of disadvantages or complications. For example, the prolonged surgical duration from the tracker setting and registration [15], the learning curve before mastering the techniques and the huge economic burden on the equipment affected the propagation and wild acceptance of computer-assisted navigation. For the later innovation, the PSBs, solved the time-consuming problem with premade manner. However, the time lag from image acquisition to manufacturing femur and tibia guides preparation and the additional charge (about \$400-900) on the patient's side prevent the wide adoption of this device in our daily practices.[16]

Based on the past experiences in navigation-assisted TKAs, the authors acknowledged the strength of this computer-assisted techniques with real-time verifications. $[17,18]$ However, the experienced surgeons can determine and execute the adequate bone cuts to similar precision even without the aid of CAN techniques. Further, basic requirement of TKAs is to create an adequate parallel space between distal femur and proximal tibia for the implantation of prostheses. Thus, it is not hard for trained surgeons to execute the mentioned tasks with their firm and steady oscillating saws according to the prudent preoperative plan as well as the reliable verifying principles during surgery.

Compared to the literature reporting CAN-TKAs with retained implants $[5,9,14,19-21]$, our study is the first to specifically present the usefulness of free-hand techniques of proximal tibia and distal femur cuts in these complicated TKAs with retained implant. The outcomes in our series prove the mentioned ideas useful in the presence of retained hardware around the knee. Even though the initial deformity of the lower limb varies from $25 \rrbracket$ varus to $10 \rrbracket$ valgus, a correct alignment with a deviation less than 3 degrees was achieved in all cases after surgery. The mean ischemic time is 93.6 minutes, which was thought to be acceptable in complex knee arthroplasties. Mean hemoglobin drop was $3.2 \mathrm{~g} / \mathrm{dL}$, which was not inferior to routine primary TKA. [22] During hospitalization, no patients required allogenic blood transfusion or prolonged inpatient stay. No patients had wound complications, periprosthetic infection and any related readmission. This may be due to the reduced surgical trauma, perioperative blood loss by not violating medullary canal as well as the limited removal of the interfering hardware. These favorable results of current study are no inferior to those using CAN or PSB in TKA with retained hardware which also report no surgical site-specific complications in their matched cohort studies.[5, 9] Manrique et al. reported significant higher mechanical failure in TKA with retained hardware compared with matched cohort.[23] However, this condition was not observed in our

Page $4 / 11$ 
patients nor the studies with retained hardware before. In the conversion TKA series, a trend of higher mechanical failure including periprosthetic fracture and loosening was also reported. We are unable to draw a conclusion that which is mechanically more fragile between TKA with retained hardware and conversion TKA. Image evaluation before operation should be done case by case to identify hindering implants that may cause suboptimal prothesis implantation. Post-operative protocols should be adjusted if stress risers are of concern.

Some inherent limitations of this study are its retrospective nature, relative short-term outcomes and the limited sample size without control group. The mentioned EM techniques of this study had been applied in our daily practice of routine primary TKAs, but this series includes only seven cases within 5 years due to the rarity of such complicated knees. Similar conditions had been reported in Hernandez's series (1.2\%).[13] The restoration of neutral mechanical axis is believed to ensure the durability of implanted prosthesis.[24] In the present study, the authors assessed the mechanical axis and component position using the scanogram and standard lateral radiograph. Some imaging errors were reported in the condition of flexion deformity and rotation of either femur or tibia.[25] Further evaluation with computed tomography helps to solve the mentioned problems and to verify the actual alignment of the lower limb and the positions of prostheses. However, concerns about the additional expenditure and radiation exposure prevent the use of computed tomography in our routine image follow-ups.

We acknowledge that our method may not be suitable for the junior surgeons and not be ready for generalized practice. However, no publications report the result of TKA with retained implant using simple EM method with fewer cutting jigs. With preservation of medullary canal and retained implants, clinical benefits from less surgical trauma without compromising the operation time and expenditure were to be expected.

\section{Conclusions}

This study demonstrated that post-traumatic knee arthritis can be safely managed with the use of an extramedullaryreferencing method with limited hardware removal. The parallelism between the distal femoral and proximal tibial cuts along with the intra-operative verifications of limb alignment help the surgeons to reproduce the coronal alignment of mechanical axis. The encouraging results suggest that this simple but facile alternative to perform primary TKAs in patients with arthritic knee and periarticular hardware is feasible once computer-assisted devices were unavailable.

\section{Abbreviations}

TKA: total knee arthroplasty; HKA: Hip-knee-ankle; CAN: computer-assisted navigation; PSB: patient-specific block; EM: extramedullary; ED: emergency department.

\section{Declarations}

\section{Ethics approval and consent to participate}

Informed consent was obtained from all individual participants included in the study. The study was conducted according to the Helsinki Declaration (Ethical Principles for Medical Research Involving Human Subjects) and was approved by the ethics committee of National Cheng Kung University Hospital, College of Medicine, National Cheng Kung University, Taiwan.

\section{Consent for publication}

Not applicable.

\section{Availability of data and materials}

The datasets used and analyzed during the current study are available from

the corresponding author on reasonable request. 


\section{Competing interests}

The authors declare that they have no competing interests.

\section{Funding}

This research did not receive any funding.

\section{Authors' contributions}

YKT, CYY, and CWC designed the study. KAL, CYY and CWC collected the data.

YNC and $\mathrm{CHC}$ performed the statistical analysis and interpreted the results.

YKT and CWC drafted the manuscript. The final manuscript was approved by all authors.

\section{Acknowledgements}

The authors wish to thank the medical staff of the Orthopaedic Department at

National Cheng Kung University Hospital, College of Medicine, National Cheng Kung University, Taiwan.

\section{Conflicts of interest}

The authors declare that they have no conflicts of interest in relation to this article.

\section{References}

1. Weiss, N.G., et al., Total knee arthroplasty in post-traumatic arthrosis of the knee. J Arthroplasty, 2003. 18(3 Suppl 1): p. 236.

2. Papadopoulos, E.C., et al., Total knee arthroplasty following prior distal femoral fracture. Knee, 2002. 9(4): p. 267-74.

3. Saleh, H., et al., Total knee arthroplasty for treatment of post-traumatic arthritis: Systematic review. World J Orthop, 2016. 7(9): p. 584-91.

4. Bergen, M.A., et al., Conversion Total Knee Arthroplasty: A Distinct Surgical Procedure With Increased Resource Utilization. J Arthroplasty, 2019. 34(7S): p. S114-S120.

5. Manzotti, A., et al., Post traumatic knee arthritis: navigated total knee replacement without hardware removal. Knee, 2014. 21(1): p. 290-4.

6. Kreitz, T.M., et al., A Current Procedural Terminology Code for "Knee Conversion" Is Needed to Account for the Additional Surgical Time Required Compared to Total Knee Arthroplasty. J Arthroplasty, 2017. 32(1): p. 20-23.

7. Lizaur-Utrilla, A., et al., Total Knee Arthroplasty for Osteoarthritis Secondary to Fracture of the Tibial Plateau. A Prospective Matched Cohort Study. J Arthroplasty, 2015. 30(8): p. 1328-32.

8. Ries, M.D., Prophylactic intramedullary femoral rodding during total knee arthroplasty with simultaneous femoral plate removal. J Arthroplasty, 1998. 13(6): p. 718-21.

9. Vaishya, R., V. Vijay, and A.K. Agarwal, Total Knee Arthroplasty Using Patient-specific Blocks after Prior Femoral Fracture without Hardware Removal. Indian J Orthop, 2018. 52(2): p. 154-160.

10. Jones, C.W. and S.A. Jerabek, Current Role of Computer Navigation in Total Knee Arthroplasty. J Arthroplasty, 2018. 33(7): p. 1989-1993.

11. Whiteside, L.A. and J. Arima, The anteroposterior axis for femoral rotational alignment in valgus total knee arthroplasty. Clin Orthop Relat Res, 1995(321): p. 168-72.

12. Insall, J.N., et al., Rationale of the Knee Society clinical rating system. Clin Orthop Relat Res, 1989(248): p. 13-4. 
13. Hernandez-Vaquero, D., A. Suarez-Vazquez, and S. Iglesias-Fernandez, Computer-assisted navigation in total knee arthroplasty without femoral hardware removal. Acta Chir Orthop Traumatol Cech, 2012. 79(4): p. 331-24.

14. Lin, S.Y., et al., Computer-navigated minimally invasive total knee arthroplasty for patients with retained implants in the femur. Kaohsiung J Med Sci, 2014. 30(8): p. 415-21.

15. Jenny, J.Y., et al., Consistency of implantation of a total knee arthroplasty with a non-image-based navigation system: a case-control study of 235 cases compared with 235 conventionally implanted prostheses. J Arthroplasty, 2005. 20(7): p. 832-9.

16. Stronach, B.M., et al., Patient-specific total knee arthroplasty required frequent surgeon-directed changes. Clin Orthop Relat Res, 2013. 471(1): p. 169-74.

17. Chang, C.W. and C.Y. Yang, Kinematic navigation in total knee replacement-experience from the first 50 cases. J Formos Med Assoc, 2006. 105(6): p. 468-74.

18. Chang, C.W., et al., Kinematic analysis of leg alignment during conventional versus navigated total knee arthroplasty: initial results of a prospective study. Kaohsiung J Med Sci, 2012. 28(9): p. 484-9.

19. Kim, K.K., et al., Navigation-assisted total knee arthroplasty for the knee retaining femoral intramedullary nail, and distal femoral plate and screws. Clin Orthop Surg, 2011. 3(1): p. 77-80.

20. Manzotti, A., et al., Computer-assisted total knee arthroplasty after prior femoral fracture without hardware removal. Orthopedics, 2012. 35(10 Suppl): p. 34-9.

21. Tigani, D., et al., Computer-assisted surgery as indication of choice: total knee arthroplasty in case of retained hardware or extra-articular deformity. Int Orthop, 2012. 36(7): p. 1379-85.

22. Pierson, J.L., T.J. Hannon, and D.R. Earles, A blood-conservation algorithm to reduce blood transfusions after total hip and knee arthroplasty. J Bone Joint Surg Am, 2004. 86(7): p. 1512-8.

23. Manrique, J., et al., Total Knee Arthroplasty in Patients with Retention of Prior Hardware Material: What is the Outcome? Arch Bone Jt Surg, 2018. 6(1): p. 23-26.

24. Ritter, M.A., et al., Postoperative alignment of total knee replacement. Its effect on survival. Clin Orthop Relat Res, 1994(299): p. 153-6.

25. Matziolis, G., et al., A prospective, randomized study of computer-assisted and conventional total knee arthroplasty. Threedimensional evaluation of implant alignment and rotation. J Bone Joint Surg Am, 2007. 89(2): p. 236-43.

\section{Tables}

\begin{tabular}{|lllllllll|}
\hline \multicolumn{1}{|l|}{ Table 1 patient demographic and clinical datas } \\
Patient No. & 1 & 2 & 3 & 4 & 5 & 6 & 7 & mean \\
\hline Gender & F & F & F & M & F & F & M & \\
\cline { 1 - 3 } & 67 & 72 & 60 & 68 & 69 & 57 & 76 & 67 \\
\hline Follow-up (months) & 16.4 & 21.6 & 34 & 10.2 & 7.4 & 3.9 & 15.7 & 15.6 \\
\hline Mean Hb drop (g/dL) & 2.6 & 3.2 & 3.6 & 3.6 & 1.6 & 3.2 & 4.9 & $3.2 \pm 1.0$ \\
\hline hospital stay(day) & 6 & 7 & 8 & 6 & 6 & 6 & 6 & $6.4 \pm 0.8$ \\
\hline Ischemic time(mins) & 57 & 78 & 96 & 56 & 126 & 132 & 110 & $93.6 \pm 31.1$ \\
\hline
\end{tabular}




\begin{tabular}{|c|c|c|c|c|c|c|c|c|c|}
\hline \multirow[t]{2}{*}{ Case } & \multirow{2}{*}{$\begin{array}{l}\text { Retained } \\
\text { implant }\end{array}$} & \multicolumn{4}{|c|}{ Pre-operative } & \multicolumn{4}{|c|}{ Post-operative } \\
\hline & & HKA angle & $\begin{array}{l}\text { Knee } \\
\text { score }\end{array}$ & $\begin{array}{l}\text { Function } \\
\text { score }\end{array}$ & ROM(口) & $\begin{array}{l}\text { HKA } \\
\text { angle }\end{array}$ & $\begin{array}{l}\text { Knee } \\
\text { score }\end{array}$ & $\begin{array}{l}\text { Function } \\
\text { score }\end{array}$ & ROM(口) \\
\hline 1 & $\begin{array}{l}\text { Tibia } \\
\text { interlocking } \\
\text { nail }\end{array}$ & 170 & 56 & 45 & 100 & 178 & 90 & 90 & 120 \\
\hline 2 & $\begin{array}{l}\text { Femur } \\
\text { retrograde } \\
\text { nail }\end{array}$ & 155 & 50 & 45 & 95 & 179 & 89 & 85 & 110 \\
\hline 3 & $\begin{array}{l}\text { proximal } \\
\text { tibia plate }\end{array}$ & 176 & 62 & 50 & 105 & 182 & 93 & 95 & 120 \\
\hline 4 & $\begin{array}{l}\text { Distal } \\
\text { femur DCS }\end{array}$ & 171 & 54 & 40 & 85 & 182 & 90 & 90 & 110 \\
\hline 5 & $\begin{array}{l}\text { Distal } \\
\text { femur and } \\
\text { proximal } \\
\text { tibia plate }\end{array}$ & 191 & 64 & 55 & 95 & 178 & 89 & 90 & 110 \\
\hline 6 & $\begin{array}{l}\text { femur bone } \\
\text { staples }\end{array}$ & 170 & 48 & 35 & 90 & 180 & 83 & 80 & 105 \\
\hline 7 & $\begin{array}{l}\text { Femur and } \\
\text { tibia } \\
\text { staples }\end{array}$ & 168 & 45 & 40 & 100 & 177 & 90 & 85 & 115 \\
\hline Mean & & $171.6 \pm 10.7$ & $54.1 \pm 7.1$ & $44.3 \pm 6.7$ & $95.7 \pm 6.7$ & $179.4 \pm 2.0$ & $89.1 \pm 3.0$ & $87.9 \pm 4.9$ & $112.9 \pm 5.7$ \\
\hline
\end{tabular}

\section{Figures}



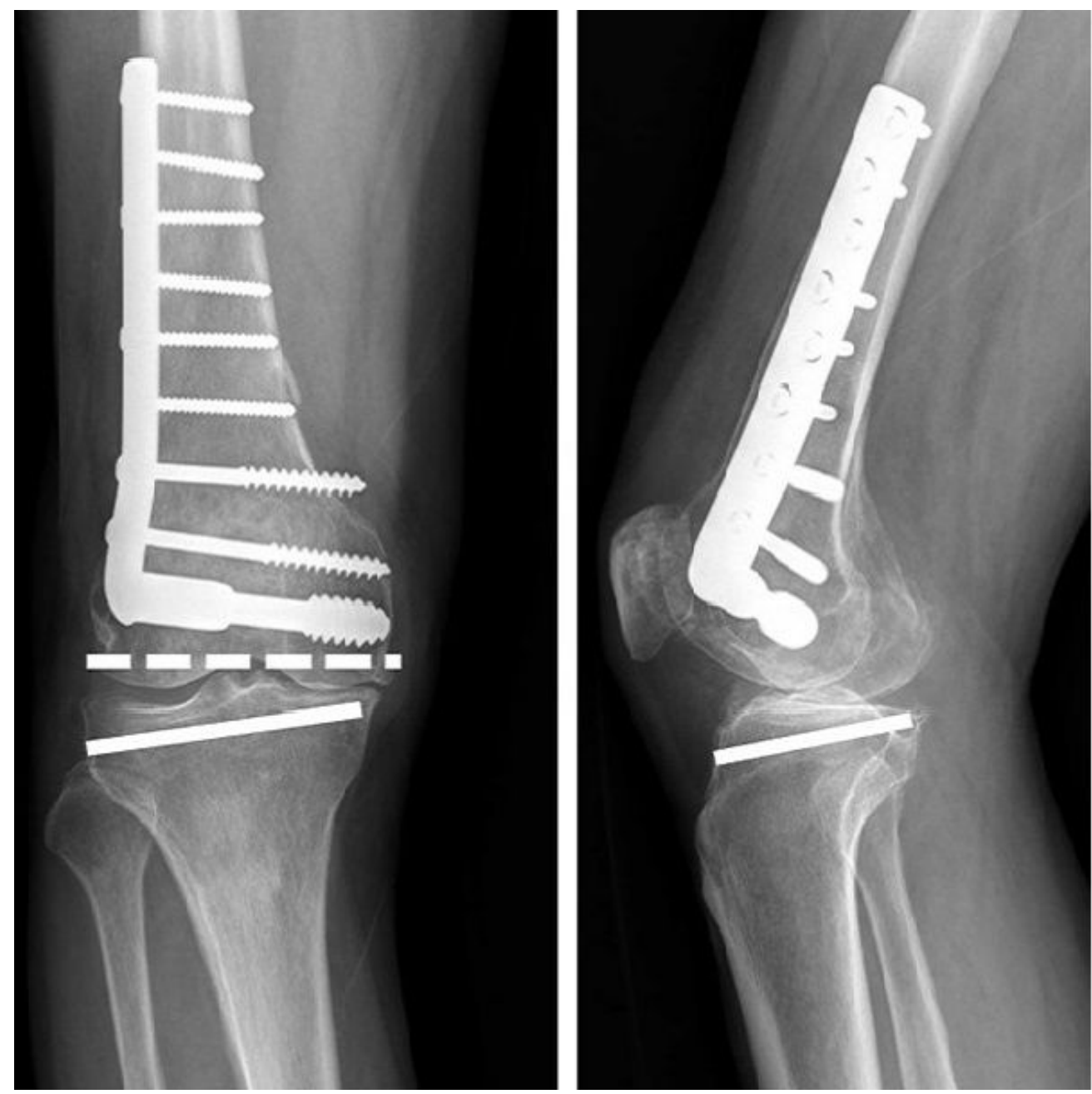

\section{Figure 1}

Pre-operative planning of proximal tibia and distal femur axial cut. Also evaluate the need of hardware removal.

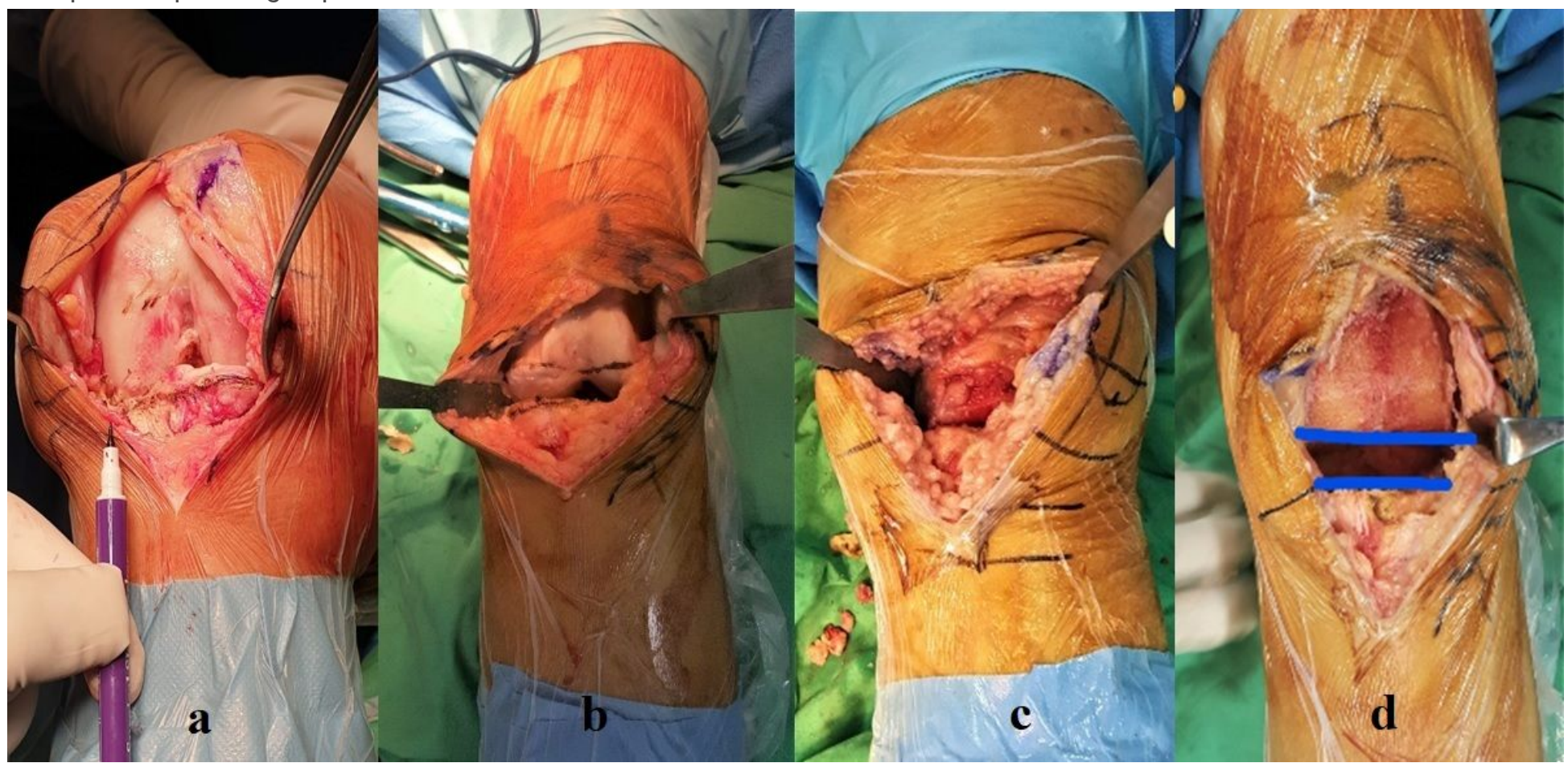

Figure 2 
(A) Horizontal line referencing tibia drawn by electrocautery. (B) Parallel line of femur by cauterization referencing proximal tibia cut. (C) Parallelism verified under knee extension. (D) Parallelism verified under knee traction.

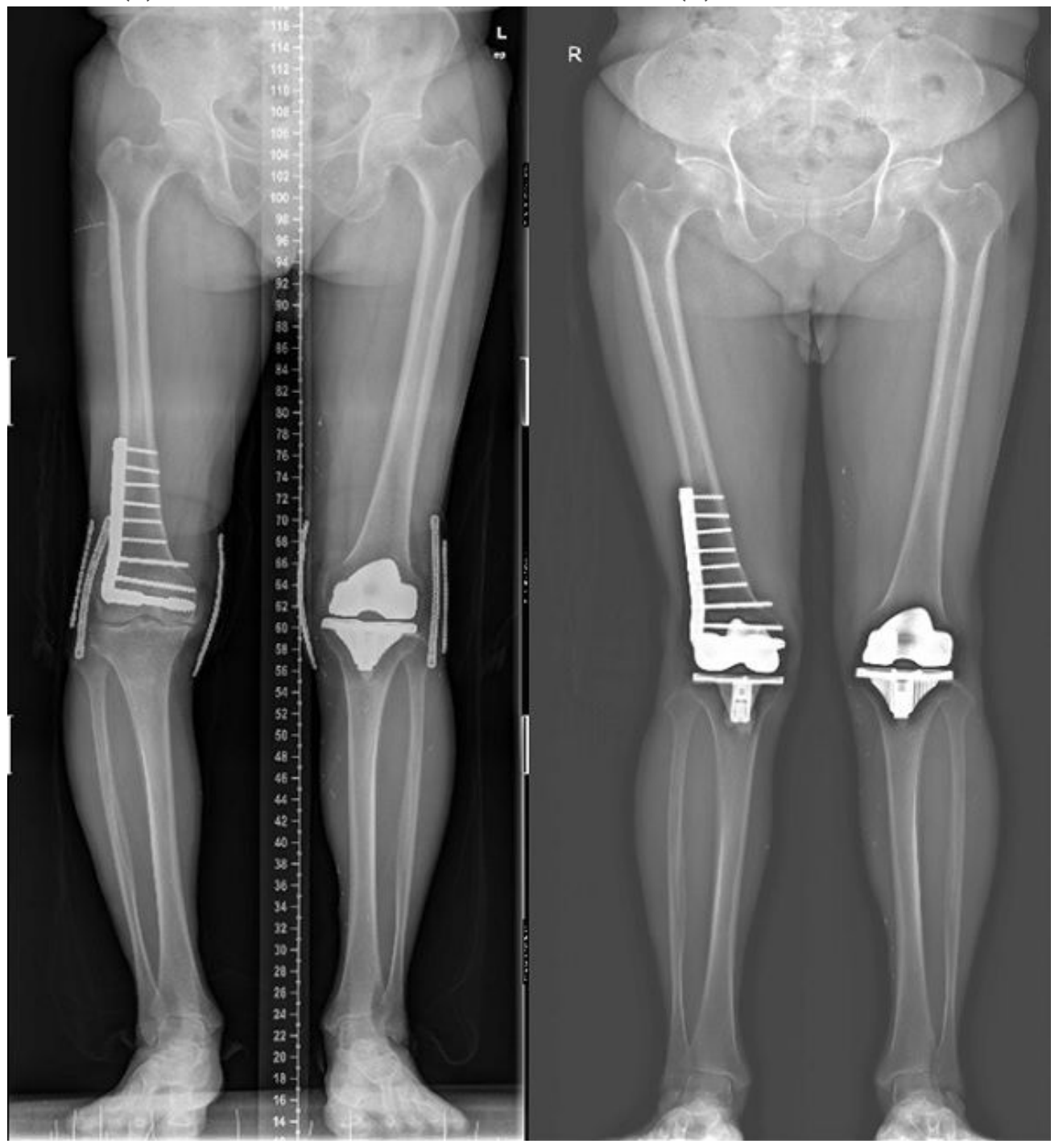

\section{Figure 3}

A 68 year-old male suffered a post-traumatic knee arthritis with a varus deformity. Primary total knee arthroplasty finished without removal of hardware. 


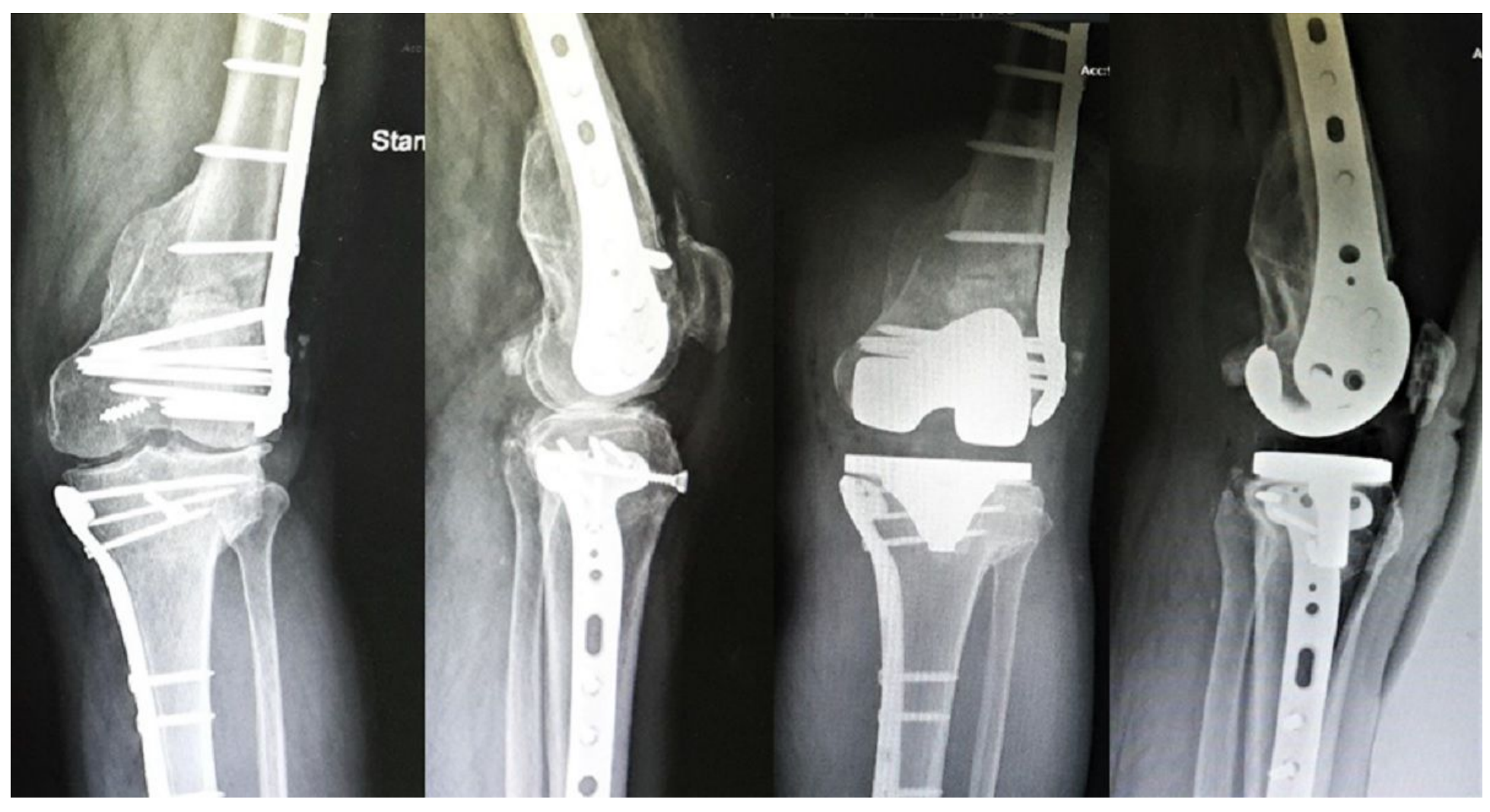

Figure 4

A 69 year-old lady suffered a post-traumatic knee arthritis with a valgus deformity. Primary total knee arthroplasty with retained hardware after limited removal of interfering screws.

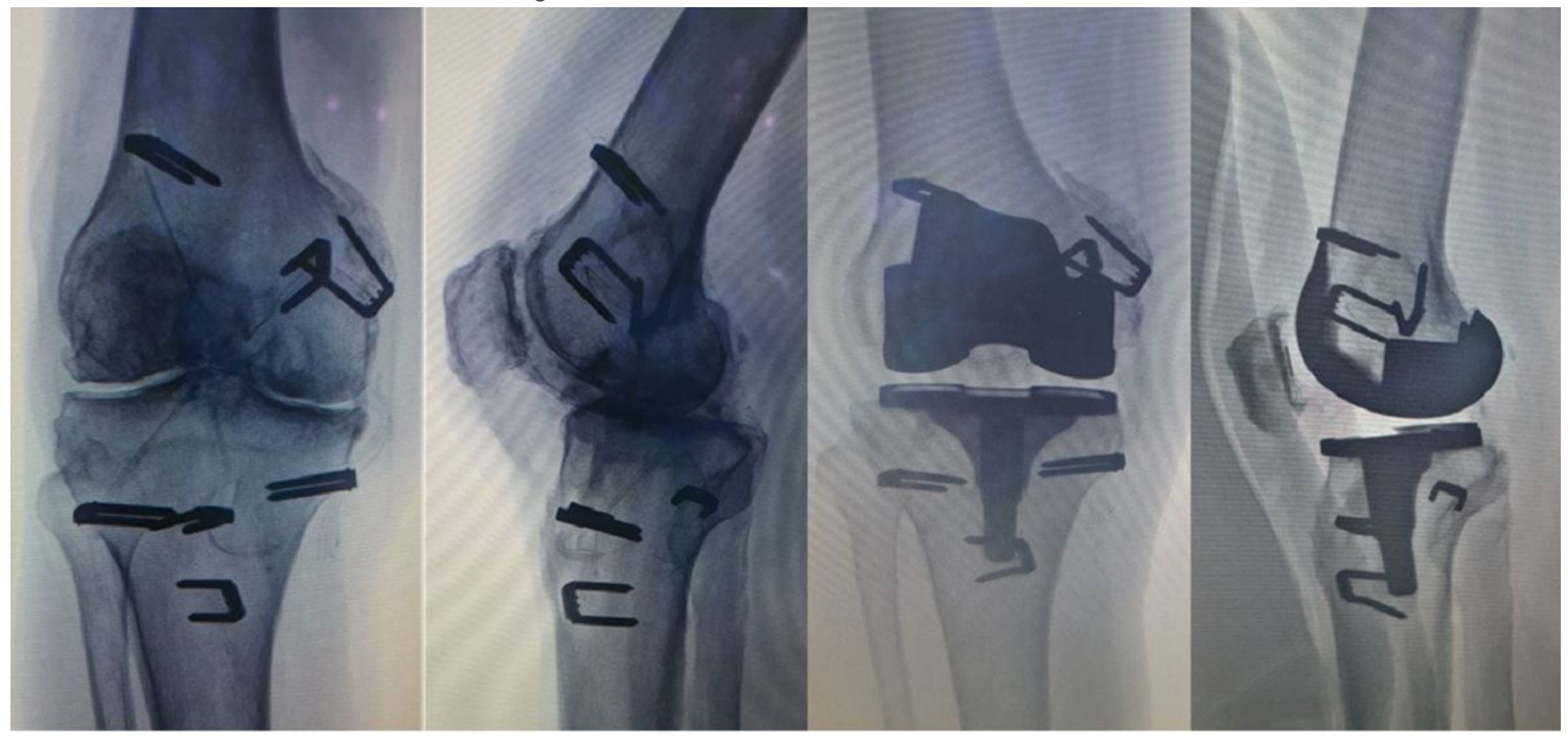

\section{Figure 5}

A 76 year-old man underwent a three-ligament reconstruction 30 years ago. Primary total knee arthroplasty with retained hardware after limited removal of one staple. 